\title{
Exploring relationships between gas dispersion parameters and differential pressure fluctuations in a column flotation
}

\author{
Xiangning Bu ${ }^{1, *}$, Shaoqi Zhou ${ }^{1}$, Meng $\operatorname{Sun}^{2}$, Muidh Alheshibri ${ }^{3,4}$, Md. ShakhaoathKhan ${ }^{5}$, \\ Guangyuan Xie ${ }^{1}$, Saeed Chehreh Chelgani6,*
}

${ }^{1}$ Key Laboratory of Coal Processing and Efficient Utilization of Ministry of Education, School of Chemical Engineering and Technology, China University of Mining and Technology, Xuzhou, Jiangsu 221116, China;

${ }^{2}$ Fengxian Power Supply Co., Ltd., State Grid Jiangsu Electric Power Co., Ltd., Fengxian, Jiangsu 221700, China;

${ }^{3}$ Department of Basic Science, Deanship of Preparatory Year and Supporting Studies, Imam Abdulrahman Bin Faisal University, P.O. Box 1982, Dammam 31441, Saudi Arabia;

${ }^{4}$ Basic \& Applied Scientific Research Center, Imam Abdulrahman Bin Faisal University, P.O. Box 1982, Dammam 31441, Saudi Arabia.

${ }^{5}$ ARC Research Hub for Computational Particle Technology, Department of Chemical Engineering, Monash University, Clayton, VIC, 3800, Australia

${ }^{6}$ Minerals and Metallurgical Engineering, Dept. of Civil, Environmental and Natural Resources Engineering, Luleå University of Technology, SE-971 87 Luleå, Sweden

$\square$ Corresponding authors:

E-mail addresses: xiangning.bu@foxmail.com, xiangning.bu@cumt.edu.cn (X., Bu); saeed.chelgani@1tu.se (S. Chehreh Chelgani) 
Table S1. The experimental results for all conditions.

\begin{tabular}{|c|c|c|c|c|c|c|c|c|}
\hline $\begin{array}{l}\text { Frother dosage } \\
(\mathrm{Cf})(\mathrm{mmol} / \mathrm{L})\end{array}$ & $\begin{array}{l}\text { Superficial water flow } \\
\text { velocity }(\mathrm{Jw})(\mathrm{cm} / \mathrm{s})\end{array}$ & $\begin{array}{c}\text { Superficial gas } \\
\text { velocity }(\mathrm{Jg})(\mathrm{cm} / \mathrm{s})\end{array}$ & $\begin{array}{l}\text { Circulating pump } \\
\text { speed (n) (rpm) }\end{array}$ & $\begin{array}{l}\text { S.D. values of pressure } \\
\text { fluctuations }(\mathrm{kPa})\end{array}$ & $\begin{array}{l}\text { Sauter bubble } \\
\text { diameter }(\mathrm{mm})\end{array}$ & $\begin{array}{c}\text { Average bubble } \\
\text { velocity }(\mathrm{ub})(\mathrm{cm} / \mathrm{s})\end{array}$ & $\begin{array}{l}\text { Reynolds number of } \\
\text { the bubbles Reb (-) }\end{array}$ & $\begin{array}{l}\text { Bubble surface } \\
\text { area flux } \mathrm{S}_{\mathrm{b}}\left(\mathrm{s}^{-1}\right)\end{array}$ \\
\hline 0.15 & 0.043 & 1.83 & 500 & 0.51 & 5.3 & 0.26 & 1378 & 20.7 \\
\hline 0.15 & 0.043 & 1.7 & 500 & 0.46 & 4.8 & 0.19 & 912 & 21.3 \\
\hline 0.15 & 0.043 & 1.44 & 500 & 0.41 & 3.8 & 0.15 & 570 & 22.7 \\
\hline 0.15 & 0.043 & 1.18 & 500 & 0.38 & 3 & 0.13 & 390 & 23.6 \\
\hline 0.15 & 0.043 & 0.88 & 500 & 0.35 & 2.6 & 0.08 & 195 & 20.3 \\
\hline 0.15 & 0.043 & 1.44 & 500 & 0.41 & 3.8 & 0.15 & 570 & 22.7 \\
\hline 0.15 & 0.096 & 1.44 & 500 & 0.41 & 3.7 & 0.16 & 581 & 23.4 \\
\hline 0.15 & 0.134 & 1.44 & 500 & 0.44 & 3.58 & 0.16 & 587 & 24.1 \\
\hline 0.15 & 0.182 & 1.44 & 500 & 0.44 & 3.53 & 0.17 & 596 & 24.5 \\
\hline 0.15 & 0.22 & 1.44 & 500 & 0.45 & 3.51 & 0.18 & 625 & 24.6 \\
\hline 0.15 & 0.043 & 1.44 & 300 & 0.53 & 6.5 & 0.32 & 2080 & 13.3 \\
\hline 0.15 & 0.043 & 1.44 & 350 & 0.48 & 6 & 0.27 & 1620 & 14.4 \\
\hline 0.15 & 0.043 & 1.44 & 400 & 0.43 & 5.7 & 0.18 & 1026 & 15.2 \\
\hline 0.15 & 0.043 & 1.44 & 450 & 0.42 & 5.1 & 0.17 & 867 & 16.9 \\
\hline 0.15 & 0.043 & 1.44 & 500 & 0.48 & 4.5 & 0.16 & 720 & 19.2 \\
\hline 0.15 & 0.043 & 1.44 & 550 & 0.5 & 4.8 & 0.19 & 912 & 18.0 \\
\hline 0.23 & 0.043 & 1.44 & 500 & 0.41 & 3.85 & 0.14 & 524 & 22.4 \\
\hline 0.15 & 0.043 & 1.44 & 500 & 0.41 & 3.8 & 0.15 & 570 & 22.7 \\
\hline 0.08 & 0.043 & 1.44 & 500 & 0.46 & 4.3 & 0.17 & 731 & 20.1 \\
\hline 0.04 & 0.043 & 1.44 & 500 & 0.48 & 5.49 & 0.22 & 1208 & 15.7 \\
\hline
\end{tabular}

